\title{
Study of the Range of Applicability of the Caustics Optical Set-Up for the Stress Intensity Factor Evaluation
}

\author{
George A. Papadopoulos* \\ Department of Engineering Science, Section of Mechanics, National Technical University of Athens, GR-157 73, \\ Zografou campus, Athens, Greece
}

\begin{abstract}
An experimental set-up of the method of caustics was proposed for the accurate evaluation of stress intensity factor. The stress intensity factor evaluation was based on a new formula which was based on the caustic shape area. The new formula was based on the shape area of the caustics. The stress optical constants were evaluated according to the new formula of the stress intensity factor. The stress optical constants can be calculated by the shape area of the caustics. For simple and accurate evaluation of the stress intensity factor, the caustic formed by the convergent light beams (caustic (f)) was used. For this caustic, the stress optical constant is $c_{f}=v / E$, where $v$ is the Poisson's ratio and $E$ is the modulus of elasticity of the material. The Poisson's ratio and the modulus of elasticity were evaluated by the method of strain-gauges. The stress intensity factor must be independent on the $z_{0}\left(z_{0}\right.$ is the distance between reference plane and specimen) and the magnification ratio $\lambda_{m}\left(\lambda_{m}\right.$ is dependent on the $z_{0}$ and $\left.z_{i}\right)$. Experiments have shown that the stress intensity factor was changed for deference $z_{0}$ and $\lambda_{m}$ (deference $z_{i}$ ). The accurate value of the stress intensity factor is that which is closed to theoretical one. So, for a proper experimental set-up (combination of the $z_{0}$ and $z_{i}$ ) the evaluating stress intensity factor values are accurate and independent of the $z_{0}$ and $z_{i}$.
\end{abstract}

Keywords: Caustics shape area, crack problems, improvement method of caustics, optical set-up, stress intensity factors.

\section{INTRODUCTION}

The optical method of caustics $[1,2]$ has been shown to be very effective for the determination of the characteristic parameters of singular elastic fields, where the corresponding stress parameters in these particular regions are governed by singularities which render the solution of the difficult problem, if not impossible, by conventional stress analysis methods. The difficulty arises mainly from the fact that the highly strained region at the singularity is very small and therefore, the information gathered by classical experimental methods is rather vague and inaccurate.

According to the method of reflected caustics, a light beam impinges in the immediate vicinity of the singularity and is reflected from the front and rear faces of the plate (for the case of transparent materials). The reflected rays are collected along a singular surface, which is strongly illuminated. A reference screen, placed at some distance from the plate, intersects this surface and yields a singular curve, the caustic, which is, for all cases studied up to now, a generalized epicycloid. In this way, and by applying simple laws of geometric optics, that is the reflection laws, the singular stress field is transformed into an optical singularity, the caustic, which yields all the necessary information for evaluation of the stress singularity.

*Address correspondence to this author at the Department of Engineering Science, Section of Mechanics, National Technical University of Athens, GR-157 73, Zografou campus, Athens, Greece;

Tel: +30 2107721228; Fax: +30 7721302; E-mail: gpad@central.ntua.gr
The method of reflected caustics was used for the solution of several elasticity problems of particular interest in engineering applications. The method was applied primarily to cases with singularities, such as those appearing in cracked plates under plane stress conditions; to contact problems; and to problems of multiwedges (composite materials). However, the method works equally satisfactorily in elastic problems with any type of stress concentration, not necessarily including singularities. In such cases the caustic is generated from a deformed boundary instead of from a singular curve in the interior of the plate (initial curve). For a review of all these applications the reader is referred to a review papers [3-10].

The method of caustics was theoretically developed for elastic homogeneous and isotropic materials. For virtual materials, the caustic shape is dependent on the material behavior at crack tip, at the board of which, is defined by the initial curve. Also, the caustic shape is dependent on the plastic zone, core region, which is developed at crack tip because of Poisson's phenomenon. The shape of the caustic can be changed by the specimen surface curvature $[11,12]$.

The experimental method of caustics was applied to evaluate the stress intensity factors by the caustic diameters. Then, a new formula for the evaluation of the stress intensity factor which was based on the caustic area, was applied [10]. By this formula, a new approach to evaluate the stress optical constants [13]. Recent work [14-16] has demonstrated that the method of caustics can be used to study the plasticity around the crack tip. Surface properties are changed after fatigue and a permanent plastic zone at the crack-tip is remained [17-19]. 
The present work illuminates the complicated problem of the stress intensity factors evaluation by the caustic diameters which are dependent on the experimental set-up. This problem was solved by the use of the caustic area and a proper experimental set-up for the evaluation of the accurate stress intensity factors at the crack tip.

\section{METHOD OF REFLECTED CAUSTICS}

The optical method of caustics is able to transform the stress singularity into an optical singularity, using the reflection laws of geometric. For divergent or convergent light beam the reflected light rays from front, (f) and rear, (r), face of the plate form two caustics, the caustic (f) and the caustic (r). The experimental set-up is appeared in Fig. (1). For a cracked isotropic elastic specimen, the parametric equations of the two caustics are [8]:

$X_{r, f}=\lambda_{m} r_{o}\left(\cos \theta \pm \frac{2}{3} \cos \frac{3 \theta}{2}\right)$

$Y_{r, f}=\lambda_{m} r_{o}\left(\sin \theta \pm \frac{2}{3} \sin \frac{3 \theta}{2}\right)$

where $r_{o}$ is the radius of initial curve of the caustics. This radius is given by:

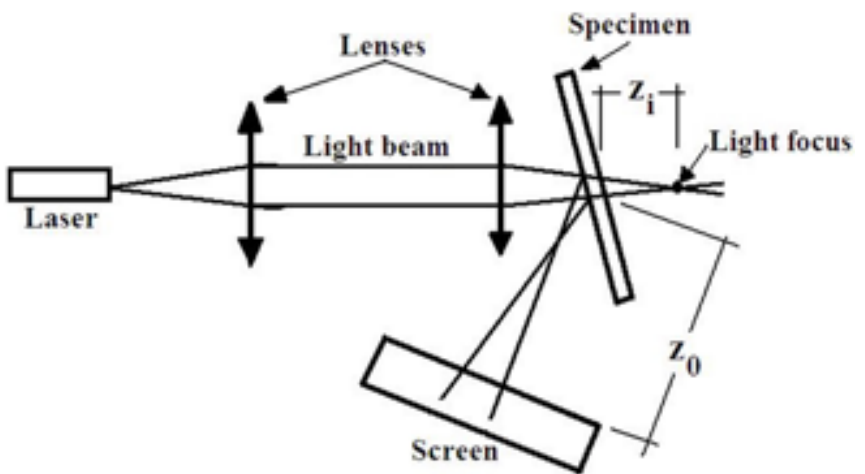

Fig. (1). Experimental optical set-up.

$r_{o}=\left(\frac{3}{2} C_{r, f}\right)^{2 / 5} \quad$ with $\quad C_{r, f}=\frac{\varepsilon z_{o} d c_{r, f} K_{I}}{\lambda_{m} \sqrt{2 \pi}}$

with:

$\lambda_{m}=\frac{z_{o} \pm z_{i}}{z_{i}}$

where $z_{o}$ is the distance between specimen and reference plane (Fig. 1), $d$ is the thickness of the specimen, $\lambda_{m}$ is the magnification ratio of the experimental set-up, $z_{i}$ is the distance between specimen and light beam focus (Fig. 1), $\varepsilon=2$ for caustic (r) and $\varepsilon=1$ for caustic (f) and $K_{I}$ is the stress intensity factor for the mode-I stress state. The $c_{r}$ and $c_{f}$ are the stressoptical constants of the material. The stress-optical constant $c_{f}$ is given by:

$c_{f}=\frac{v}{E}$

where $v$ is the Poisson's ratio and $E$ is the modulus of elasticity of the material.
The experimental stress intensity factor $K_{I}$ is estimated by the relation [8]:

$K_{I}=\frac{2 \sqrt{2 \pi}}{3 \varepsilon z_{o} d \lambda_{m}^{3 / 2} c_{r, f}}\left(\frac{D_{t, l}}{\delta_{t, l}}\right)^{5 / 2}=1.67 C\left(\frac{D_{t, l}}{\delta_{t, l}}\right)^{5 / 2}$

with

$$
C=\frac{1}{\varepsilon z_{o} d \lambda_{m}^{3 / 2} c_{r, f}}
$$

where $D_{t, l}$ are the maximum diameters of the caustics ( $D_{t}$ transverse and $D_{l}$ longitudinal) and $\delta_{t, l}$ are the correction factors with:

$\delta_{t}=3.1702$ and $\delta_{l}=3.00$

If the caustic is about circle (Fig. 2), (theoretical caustic), the diameters ratio is:

$\frac{D_{t}}{D_{l}}=\frac{\delta_{t}}{\delta_{l}}=1.056$

Then, the $K_{I}$ values are: $K_{I\left(D_{t}\right)}=K_{I\left(D_{l}\right)}$. If the caustic becomes oval (Fig. 2), (experimental caustic), $\frac{D_{t}}{D_{l}} \neq 1.056$, the $K_{I}$ values are different, $K_{I\left(D_{t}\right)} \neq K_{I\left(D_{l}\right)}$. In this state, the relation between the values of the $K_{I}$ is:

$$
K_{I\left(D_{t}\right)}=\left(\frac{D_{t} / D_{l}}{1.056}\right)^{5 / 2} K_{I\left(D_{l}\right)}
$$

Fig. (2) illustrates the experimental caustics at crack tip for crack length $\alpha=0.028 \mathrm{~m}, 0.040 \mathrm{~m}, 0.056 \mathrm{~m}$ and $0.069 \mathrm{~m}$, respectively and for the same stress $\sigma=2.42 \mathrm{MPa}$.

The flat specimens were made of Lexan (PCBA) with free length $L=0.250 \mathrm{~m}$, width $w=0.100 \mathrm{~m}$, and thickness $d=0.003 \mathrm{~m}$.

Because the shape of caustic is about ellipse, its area is given by the relation:

$A=\frac{\pi}{4} D_{t} D_{l}$

According to caustic theory [8] the diameters of the caustic are:

$D_{t}=3.1702 \lambda_{m} r_{0}, \quad D_{l}=3.00 \lambda_{m} r_{0}$

Then, from Eqs. $(3,6,10,11)$, the stress intensity factor $K_{I}$ becomes:

$K_{I}=0.1358 C A^{5 / 4}$

Then, the energy release rate or the $\mathrm{J}$ integral is given by the relation:

$G_{I}=J_{I}=\frac{K_{I}^{2}}{E}=\frac{0.0184 C^{2} A^{5 / 2}}{E}$, for plane stress state

Fig. (3) illustrates the experimental stress intensity factors (normalized to C) calculated by the diameters $D_{t}$ and 


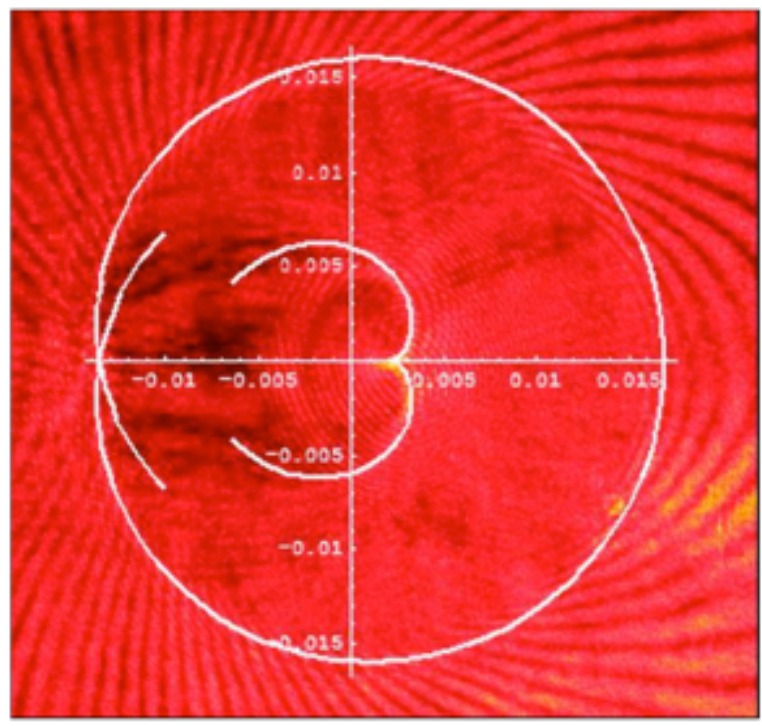

(a)

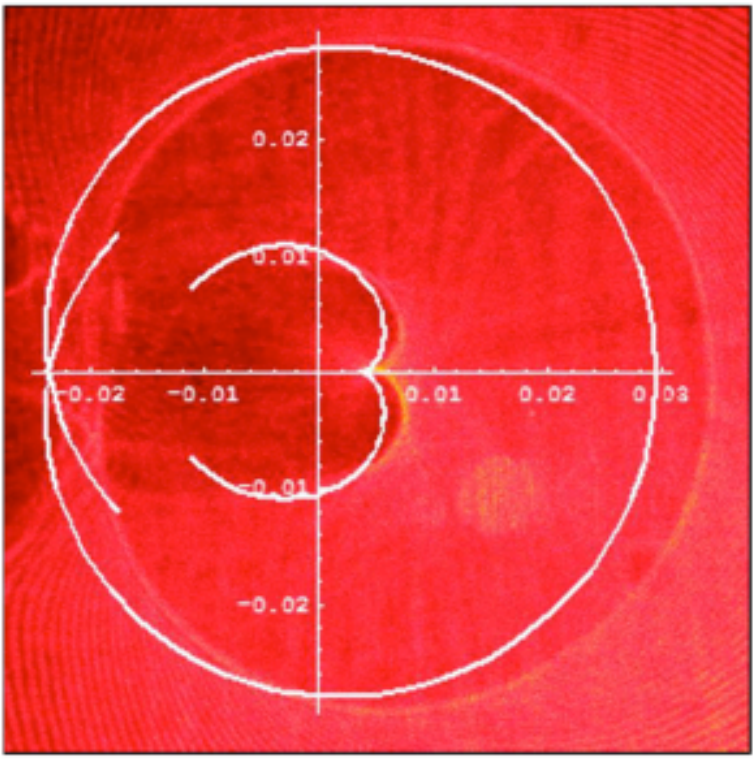

(c)

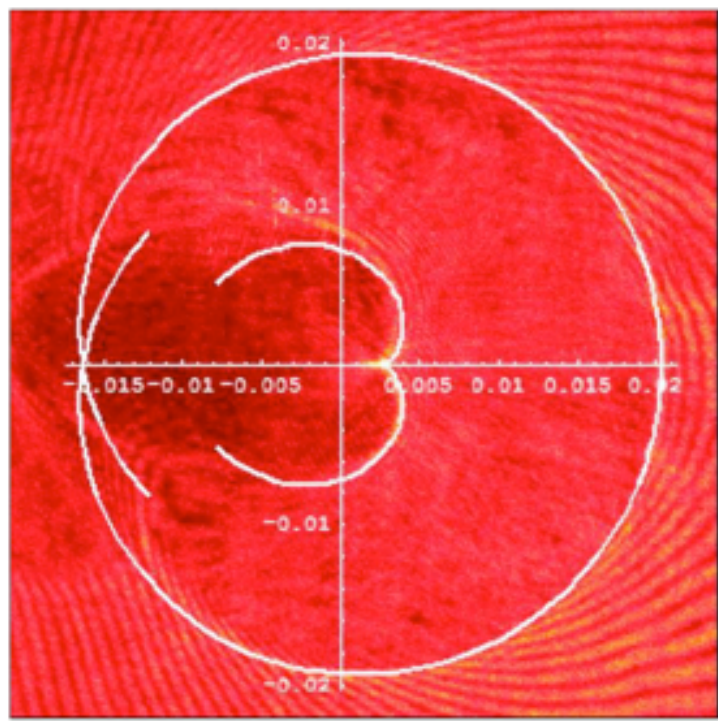

(b)

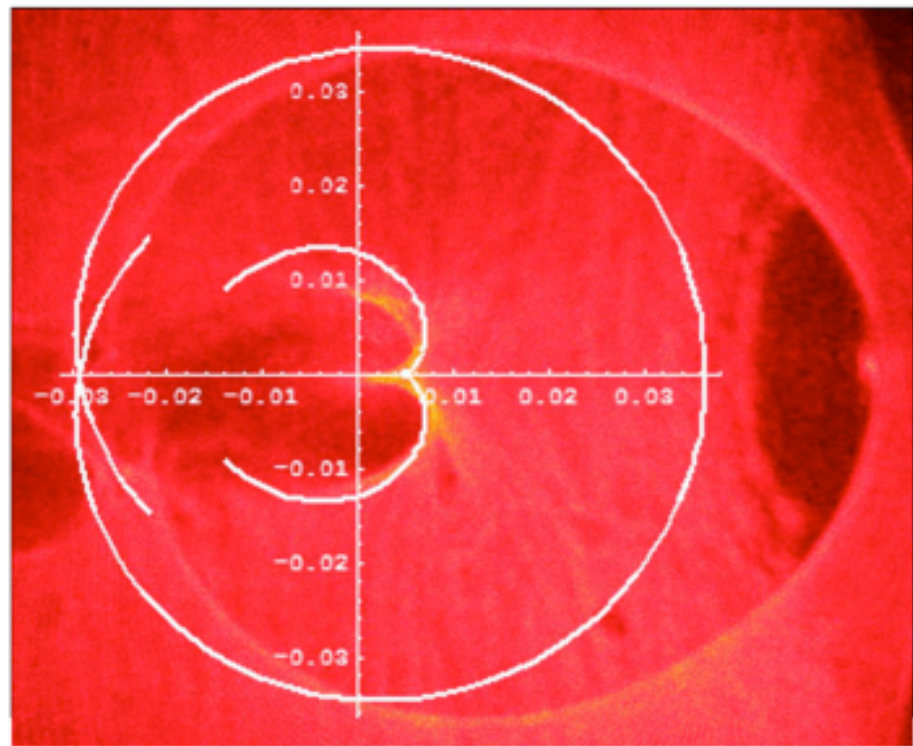

(d)

Fig. (2). Experimental caustics in Lexan (PCBA) specimen with stress $\sigma=2.42 \mathrm{MPa}$, for crack length (a) $\alpha=0.028 \mathrm{~m}$, (b) $\alpha=0.040 \mathrm{~m}$, (c) $\alpha=0.056 \mathrm{~m}$ and (d) $\alpha=0.069 \mathrm{~m}$.

$D_{\ell}$ and shape area, $A$, of the caustic versus the tensile stress for the same crack length $\alpha=0.040 \mathrm{~m}$. From this figure, it is appeared that the curve of the stress intensity factor, which was calculated by the area of the caustic, lies between the two others. From these curves a linear variation and values coincidence of the stress intensity factor up to $\sigma=12 \mathrm{MPa}$ can be observed. After this stress, a non-linear variation and values deviation can be observed. This means that the stress intensity factors values in this stress region, which were calculated with the area of the caustic, are more accurate than the others.

\section{EVALUATION OF THE STRESS OPTICAL CONSTANTS}

The $c_{r}$ and $c_{f}$ are the stress-optical constants of the material. The stress-optical constant $c_{f}$ is given by: $c_{f}=v / E$, where $v$ is the Poisson's ratio and $E$ is the modulus of elasticity of the material. The stress optical constant $c_{f}$ can be calculated according to method of [20] or by the method of strain gauge. The plotting of the two caustics for divergent light beam is illustrated in Fig. (4). The parametric equations of the caustics (r) are: 


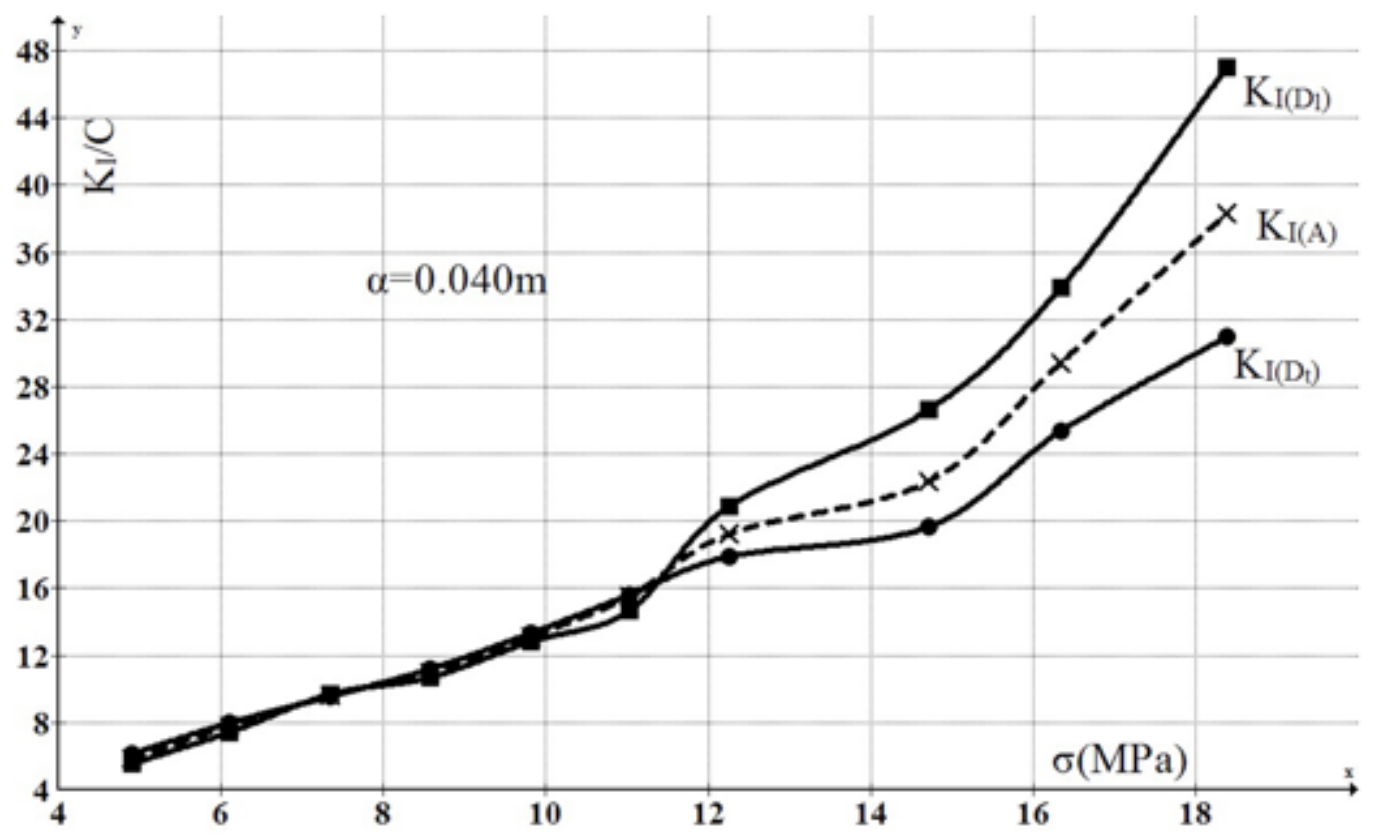

Fig. (3). Variation of the stress intensity factors calculated by the diameters, $K_{I\left(D_{t}\right)}, K_{I\left(D_{t}\right)}$ and shape area, $K_{I(A)}$, of the caustic $v s$ the crack length for the same stress.

$X_{r}=\lambda_{m} r_{o}\left(\cos \theta+\frac{2}{3} \cos \frac{3 \theta}{2}\right)$

$Y_{r}=\lambda_{m} r_{o}\left(\sin \theta+\frac{2}{3} \sin \frac{3 \theta}{2}\right)$

where $r_{o}$ is the radius of initial curve of the caustics. This radius is given by:

$r_{o}=\left(\frac{3}{2} C_{r}\right)^{2 / 5} \quad$ with $\quad C_{r}=\frac{2 z_{o} d c_{r} K_{I}}{\lambda_{m} \sqrt{2 \pi}}$

with:

$\lambda_{m}=\frac{z_{o}+z_{i}}{z_{i}}$

From Eqs. $(14,15)$, according to Fig. (4) for $\theta=0$, we get:

$c_{r}=\frac{c_{f}}{2\left[5-8.18 \Delta x / A_{(r)}^{1 / 2}\right]^{5 / 2}}$

where $\Delta x$ is the distance (BA) and $A_{(r)}$ is the area of the ( $r$ ) caustic. By the Eq. (3) the ratio of the stress-optical constants $c_{r}, c_{f}$ is estimated. Analogous relation to Eq. (17) from Fig. (5) can be written by combination of caustics (r) and (f) for convergent light beam. The parametric equations of the caustic (f) are:

$X_{f}=\lambda_{m} r_{o}\left(\cos \theta+\frac{2}{3} \cos \frac{3 \theta}{2}\right)$

$Y_{f}=\lambda_{m} r_{o}\left(\sin \theta+\frac{2}{3} \sin \frac{3 \theta}{2}\right)$ where $r_{o}$ is the radius of initial curve of the caustics. This radius is given by:

$r_{o}=\left(\frac{3}{2} C_{f}\right)^{2 / 5} \quad$ with $\quad C_{f}=\frac{z_{o} d c_{f} K_{I}}{\lambda_{m} \sqrt{2 \pi}} \quad$ and $\quad c_{f}=\frac{v}{E}$

with:

$\lambda_{m}=\frac{z_{o}-z_{i}}{z_{i}}$

From Eqs. $(18,19)$, according to Fig. (5) for $\theta=0$, we get:

$c_{r}=\frac{c_{f}}{2}\left[5-8.18 \Delta x / A_{(f)}^{1 / 2}\right]^{5 / 2}$

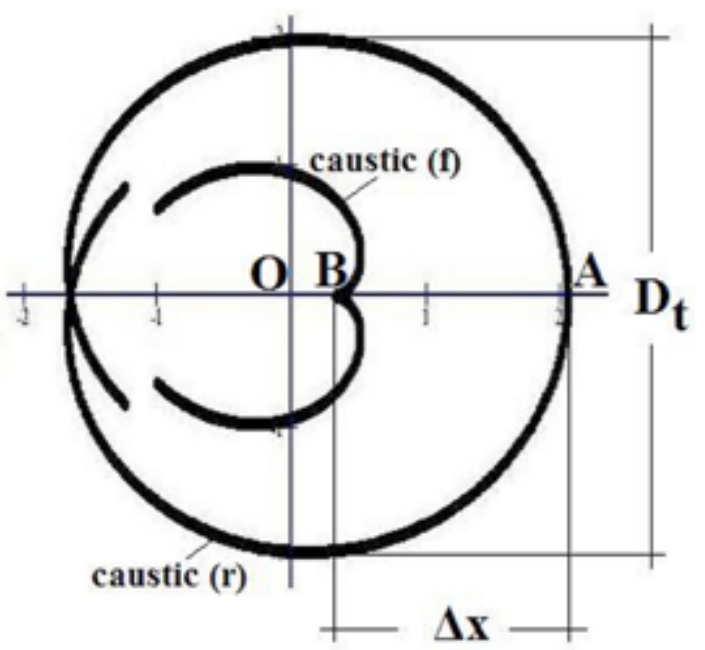

Fig. (4). Geometry of caustics (r) and (f) for divergent light beam. 


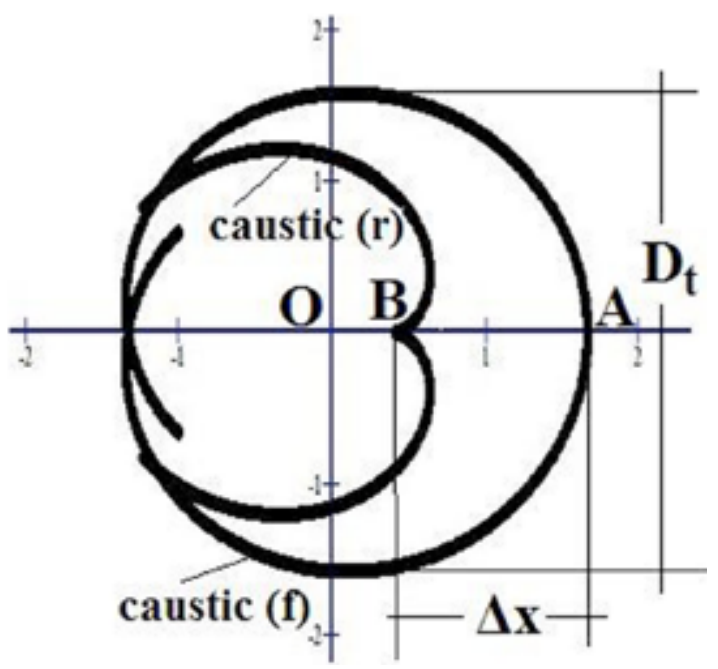

Fig. (5). Geometry of caustics (r) and (f) for convergent light beam.

The experimental stress intensity factor $K_{I}$ is estimated by the Eq. (6). From Eqs. (6 and 17), the accurate stress intensity factor can be estimated from Poisson's ratio $v$ and the modulus of elasticity $E$ or the stress-optical constant $c_{f}$ and the caustic area:

$K_{I}=\frac{0.1358}{z_{o} d \lambda_{m}^{3 / 2} c_{f}}\left(5 A_{(r)}^{1 / 2}-8.18 \Delta x\right)^{5 / 2}, \quad c_{f}=\frac{v}{E}$

For convergent light beam, the stress intensity factor is estimated by the relation:

$$
K_{I}=0.1358 C A^{5 / 4} \quad \text { with } \quad C=\frac{1}{z_{o} d \lambda_{m}^{3 / 2} c_{f}} \quad \text { and } \quad c_{f}=\frac{v}{E}
$$

\section{EXPERIMENTAL SET-UP AND ITS INFLUENCE ON THE STRESS INTENSITY FACTOR}

The stress intensity factor must be independent on the experimental set-up. In order to prove that the stress intensity factor is independent on the experimental set-up, a series of experiments were undertaken with an edge cracked specimen. The specimen was made of Plexiglas with free length $\mathrm{L}=0.020 \mathrm{~m}$, width $\mathrm{w}=0.080 \mathrm{~m}$, thickness $\mathrm{d}=0.004 \mathrm{~m}$ and crack length $\alpha=0.020 \mathrm{~m}$. The crack width was about $0.0003 \mathrm{~m}$. The parametric equations of the reflected caustics (f) which were formed by the convergent light beam, reflection from the front face of the specimen, are the Eqs. (18 and 19). The modulus of elasticity and the Poisson's ratio of the specimen materials were calculated by the method of strain-gauges. The modulus of elasticity was $E=1.54 G P a$, the Poisson's ratio was $v=0.4$ and the stress optical constant was $c_{f}=v / E=2.6 \times 10^{-10} \mathrm{~m}^{2} / N$.

The theoretical stress intensity factor was estimated by the equation:

$$
K_{I}^{t h}=1.12 \sigma \sqrt{\pi \alpha}
$$

while the experimental stress intensity factor was estimated by the relation [10]:

$$
K_{I}^{\exp }=\frac{0.1358}{z_{o} d \lambda_{m}^{3 / 2} c_{f}} A^{5 / 4} \quad \text { with } \quad c_{f}=\frac{v}{E}
$$

where $A$ is the shape area of the experimental caustics.

Three experiments were undertaken. For the first experiment, the specimen was loaded with constant tensile stress $\sigma=2.69 \mathrm{MPa}$, the $z_{i}$ was constant equals $z_{i}=0.380 \mathrm{~m}$ and the $z_{0}$ was varied from $1.18 m$ up to $2.10 m$, Fig. (1). The theoretical stress intensity factor was calculated by the Eq. (24) equals $K_{I}^{\text {th }}=0.76 \mathrm{MPam}^{1 / 2}$. A series of experimental caustics were taken and the experimental stress intensity factors were calculated by the Eq. (25). For the second experiment, the tensile stress was remained the same, the $z_{0}$ was constant equals $z_{0}=1.33 \mathrm{~m}$ and the $z_{i}$ was varied from $0.045 \mathrm{~m}$ up to $0.380 \mathrm{~m}$ (Fig. 1). The theoretical stress intensity factor was remained the same. For the third experiment, the $z_{0}$ and $z_{i}$ were constant equal $z_{0}=1.58 \mathrm{~m}$ and the $z_{i}=0.360 m$ (Fig. 1), and the tensile stress $\sigma$ was varied from $0.90 \mathrm{MPa}$ up to $4.04 \mathrm{MPa}$. The theoretical stress intensity factor was varied analogous to variation of the tensile stress.

Fig. (6) illustrates the experimental caustics (f) at crack tip for the three experiments. The experimental stress intensity factor $K_{I}^{\exp }$ was calculated by the Eq. (25). The results from the first experiment are presented in Fig. (7). Fig. (7) illustrates the variation of the experimental stress intensity factor $K_{I}^{\text {exp }}$ versus the variation of the distance $z_{0}$ for constants $\sigma$ and $z_{i}$. In the same figure the constant theoretical stress intensity factor $K_{I}^{\text {th }}$ was plotted. The experimental values of the stress intensity factor are very close to theoretical one.

The results from the second experiment are presented in Fig. (8). Fig. (8) illustrates the variation of the experimental stress intensity factor $K_{I}^{\exp }$ versus the variation of the distance $z_{i}$ for constants $\sigma$ and $z_{0}$. In the same figure the constant theoretical stress intensity factor $K_{I}^{\text {th }}$ was plotted. The experimental values of the stress intensity factor are converged to theoretical one for $z_{i}>0.20 \mathrm{~m}$.

The results from the third experiment are presented in Fig. (9). Fig. (9) illustrates the variation of the experimental and the theoretical stress intensity factors $K_{I}^{\exp }, K_{I}^{\text {th }}$ versus the variation of the tensile stress $\sigma$ for constants $z_{0}$ and $z_{i}$.

The experimental values of the stress intensity factor are converged to theoretical one for stresses $\sigma<2.5 \mathrm{MPa}$ while, it is appeared a declination for stresses $\sigma>2.5 \mathrm{MPa}$.

From Figs. $(\mathbf{7}, \mathbf{8})$, it is concluded that a proper combination of $z_{0}$ and $z_{i}$, the stress intensity factors become independent from the experimental set-up. So, for $z_{i}>0.20 m$ and for any $z_{0}$ the calculated stress intensity factors are remained constants and close to theoretical one. 


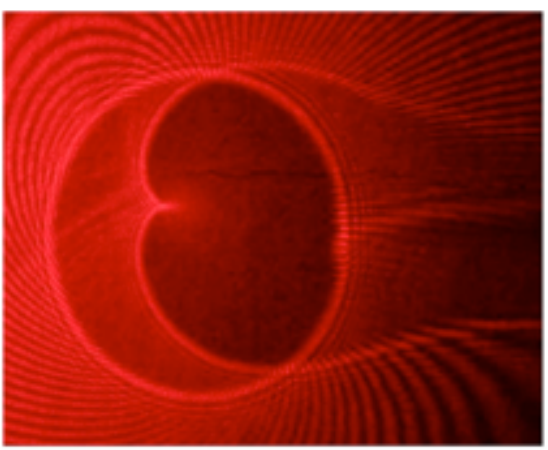

(a)

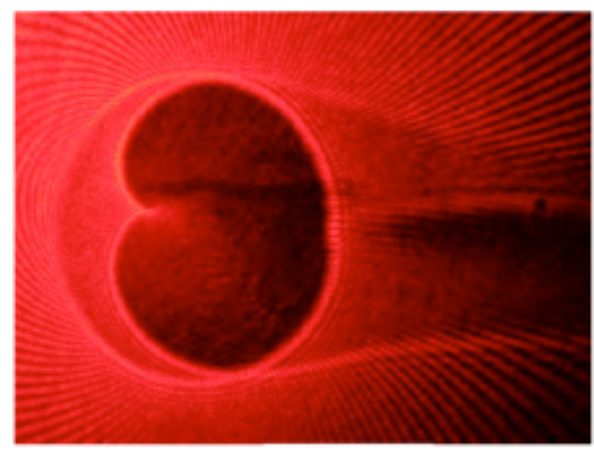

(b)

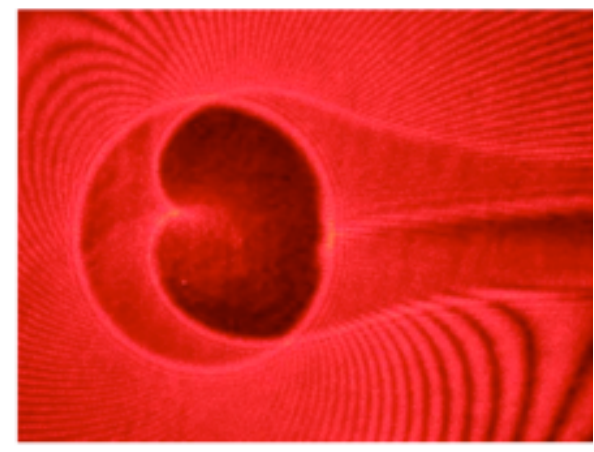

(c)

Fig. (6). Experimental caustic (f) from (a) first experiment, (b) second experiment and (c) third experiment.

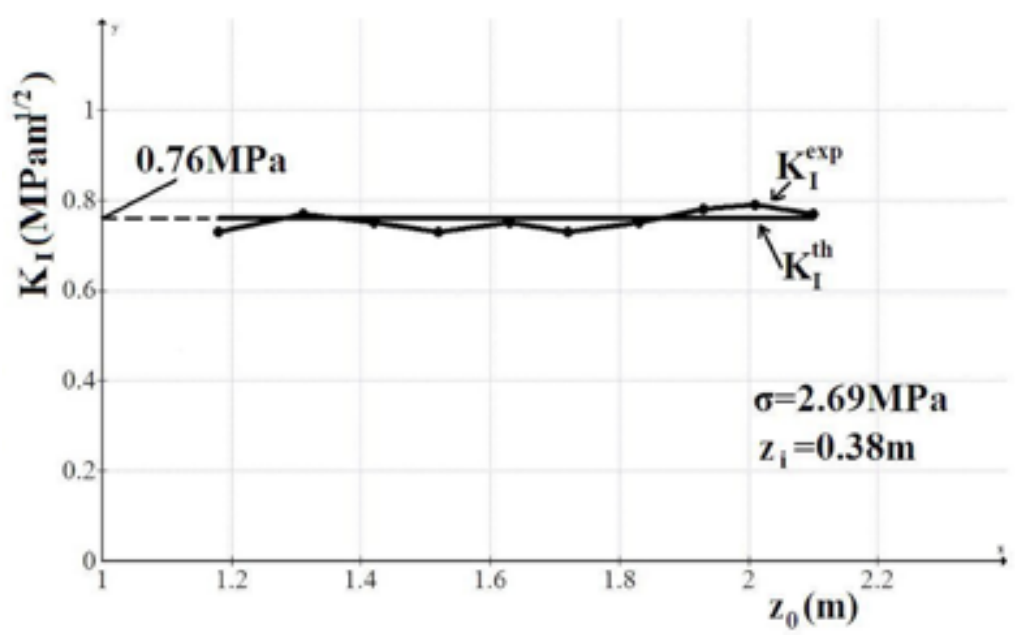

Fig. (7). Variation of the stress intensity factors $v s$ the distance $z_{0}$.

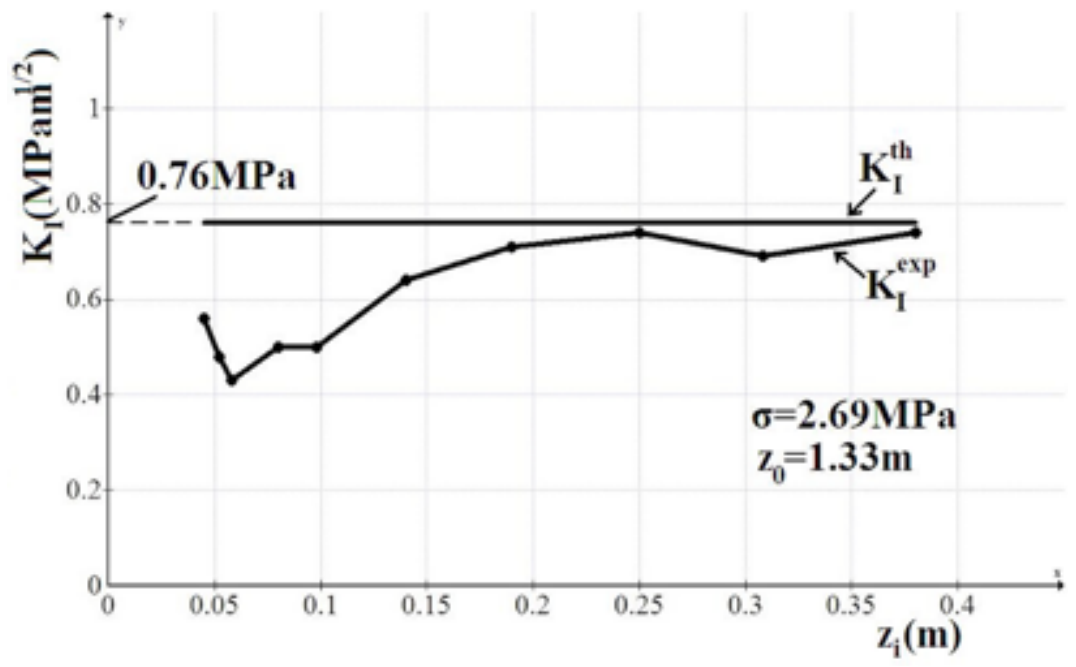

Fig. (8). Variation of the stress intensity factors $v s$ the distance $z_{i}$.

From Fig. (9), it is appeared that for stresses $\sigma>2.5 \mathrm{MPa}$ the stress intensity factors are remained smaller than the theoretical one because of plastic zones are created at crack tip which are dependent on the type of the materials.

\section{CONCLUSION}

The proposed new formula, Eq. (12), which depends on the shape area of the caustic, was given more accurate and stable values of the stress intensity factors. The stress intensity factor becomes independent from the diameters of the caustic, which were difficultly calculated if the caustic is not about circular [10]. Frequently, the stress intensity factors are dependent on the experimental set-up, mainly on $z_{0}$ and $z_{i}$. For a proper combination of $z_{0}$ and $z_{i}$, for example for $z_{i}>0.20 \mathrm{~m}$ and for any $z_{0}$, the stress intensity factors are remained constant and close to theoretical one. 


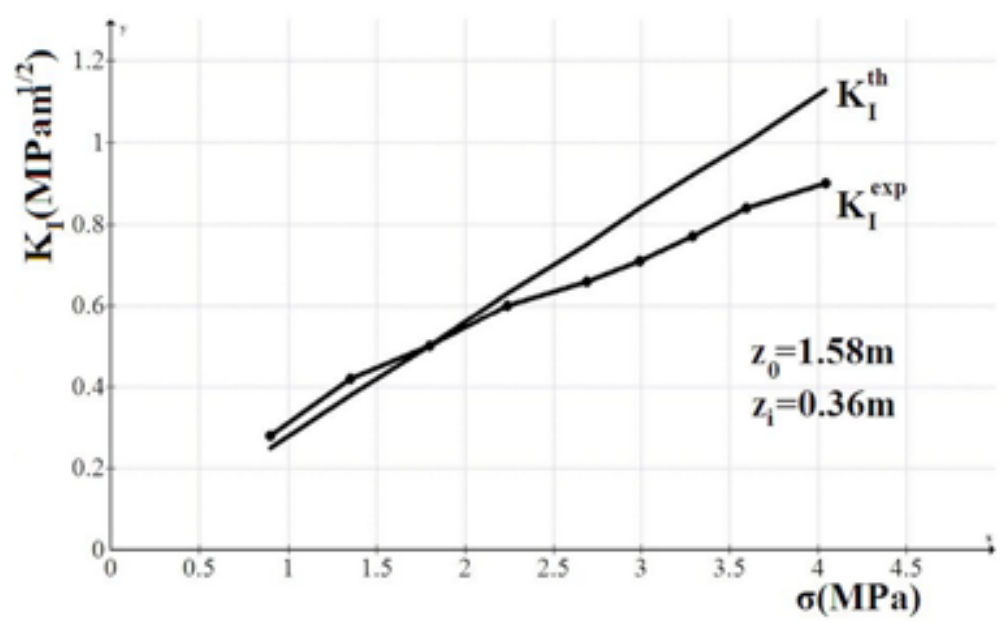

Fig. (9). Variation of the stress intensity factors $v s$ the tensile stress $\sigma$.

\section{CONFLICT OF INTEREST}

The author confirms that this article content has no conflict of interest.

\section{ACKNOWLEDGEMENTS}

Declared none.

\section{REFERENCES}

[1] P. Manogg, "Anwendung der Schattenoptik zur Untersuchung des Zerreissvorgangs von Platten," Dissertation 4/64, Universitaet Freiburg, 1964.

[2] P.S. Theocaris, "Local yielding around a crack tip in plexiglas", $J$. Appl. Mech., vol. 37, pp. 409-415, 1970.

[3] P.S. Theocaris, and E.E. Gdoutos, "The Modified Dugdale - Barenblatt model adapted to various fracture configurations in metals", Int. J. Fract., vol. 10, pp. 549-564, 1974

[4] P.S. Theocaris, and G.A. Papadopoulos, "Stress corrosion crack growth in aluminum alloys." Eng. Fract. Mech., vol. 9, pp. 781-794, 1977.

[5] G.A. Papadopoulos, "Evaluation of concentrated oblique load at the apex of a wedge by the method of caustics", Open Mech. Eng. J., vol. 6, pp. 90-99, 2012.

[6] A.J. Rosakis, C.C. Ma, and L.B. Freund, "Analysis of the optical shadow spot method for a tensile crack in a power-law hardening material", J. Appl. Mech. vol. 50, pp. 777-782, 1983.

[7] J.F. Kalthoff, In: Kobayashi AS, Ed. Handbook on Experimental Mechanics, Chap. 9, pp. 430-500, Prentice-Hal, 1987.

[8] G.A. Papadopoulos, The experimental method of caustics and the det.-criterion of Fracture, In: Fracture Mechanics, Springer-Verlag, London, 1993.
[9] G.A. Papadopoulos, "Experimental study of the load distribution in bearings by the method of caustics and the photoelasticity method", J. Strain Anal., vol. 40(4), pp. 357-365, 2005.

[10] G.A. Papadopoulos, "New formula of experimental stress intensity factor evaluation by caustics", Int. J. Fract., vol. 171, pp. 79-84, 2011.

[11] P.S. Theocaris, and C.G Thireos, "Stress intensity factors in cracked cylindrical shells under tension", Int. J. Fract., vol. 12, pp. 691703, 1976.

[12] R.A. Tomlisson, and E.A. Patterson, "The effects of surface topography on the method of caustics", Exp. Mech., vol. 39(4), pp. 335-342, 1999.

[13] B. Badalouka, and G.A. Papadopoulos, "Experimental evaluation of stress-optical constants by caustics", Int. J. Fract., vol. 171, pp. 8590, 2011.

[14] D.J. Unger, "Perfectly plastic caustics for the opening mode of fracture", Theory Appl. Fract. Mech., vol. 44, pp. 82-94, 2005.

[15] S. Hedans, O. Pop, V. Valle, and M. Cottron, "FE and experimental investigation with shadow optical method for measuring plastic zone in a ductile cracked plate", Strain, 2008. DOI:10.1111/j.14751305.2008.00594.x

[16] B.G. Badalouka, and G.A. Papadopoulos, "Experimental evaluation of the plastic zone at crack tip by caustics", Open Mech. Eng. J., vol. 6, pp. 83-89, 2012

[17] R. Avilıs, J. Albizuri, A. Rodrvguez, and L.N. Lopez de Lacalle, "Influence of low-plasticity ball burnishing on the high-cycle fatigue strength of medium carbon AISI 1045 steel", Int. J. Fatigue, vol. 55, pp. 230-244, 2013.

[18] G.A. Papadopoulos and D.A. Korovesis: "Study of fatigue internal damage of aluminum alloy." Adv. Compos. Lett., vol. 3(3), pp. 109114, 1994.

[19] G.A. Papadopoulos and D.A. Korovesis: "Study of fatigue Internal damage of nylon 6.", J. Mater. Sco., vol. 15, pp. 995-996, 1996.

This is an open access article licensed under the terms of the Creative Commons Attribution Non-Commercial License (http://creativecommons.org/licenses/ by-nc/3.0/) which permits unrestricted, non-commercial use, distribution and reproduction in any medium, provided the work is properly cited. 